\title{
S19. How to assess innovating new drugs
}

PROBLEMS WITH PLACEBO CONTROLLED STUDIES Stuart A Montgomery

St Mary's Hospital Medical School, London W2

Evidence of efficacy can only be obtained from proper placebo controlled studies in a group of patients who require treatment. The reluctance of some European investigators to withhold effective treatments and carry out placebo controlled studies means that they do not play any part in establishing whether a drug is effective. Comparator studies are useful to establish relative safety but not efficacy. When placebo controlled studies of acute treatment are undertaken in Europe the investigators appear to either select patients who do not need a drug or to provide concomitant behavioural or cognitive support which invalidates the study by producing a high placebo response rate.

The high placebo response rate in a variety of acute studies makes this design less preferred. The long term treatment studies are easier and more ethical to perform given the patients are followed up while in a well condition. The results of these studies provide unequivocal and clinically relevant evidence of the efficacy of drug.

\section{THE VALUE OF HUMAN BRAIN IMAGING FOR EARLY DRUO} TRIALS

G Sedvall, L Farde. P Karlsson, $H$ Hall and C. Halldin

Department of Clinical Neuroscience. Psychiatry and Psychology Section, Karolinska Institute and Hospital, S-171 76 Stockholm, Sweden.

There is an apparant need to expedite procedures for the clinical evaluation of innovating new drugs in psychiatry. The development of positron emission tomography and the possibility to use this technique for visualizing and quantifying drug effects on neuroreceptors and other binding sites in the brain represents a promising new strategy in this respect. Also in vitro studies on human brain tissue should be helpful in this regard since most developments regarding drug targets in the preclinical phase concern drug targets in a nonhuman environment. Recent technical developments allow the detailed evaluation of drug effects in vitro on whole human brain cryosectioned hemispheres. Such studies directly give indications for optimal drug concentrations in the brain to induce the occupancy levels of specific receptors aimed for in all regions of the human brain. These in vitro results can be verified by subsequent in vivo studies using positron emission tomography. Such studies in addition give early indications concerning human pharmacokinetics and metabolism of the drugs in question. They also supply information concerning the oral dose levels required to induce specific occupancy levels in the brain.

This potential is exemplified by a series of studies with the new sclective D1 dopamine receptor antagonist SCH 39166. This compound has been proposed to be an effective antipsychotic drug. On the basis of in vitro and PET imaging studies appropriate doses were proposed. These doses were subsequently evaluated in a clinical study in schizophrenic patients. In spite of a high D1 dopamine receptor occupancy induced by this compound no antipsychotic effect was recorded in the patients. These results indicate that selective D1 dopamine receptor blockade does not induce antipsychotic effects. Accordingly the PET studies precluded the performance of expensive dose finding studies with this compound. 


\section{NOVEL TARGETS AND STRATEGIES FOR DRUG DISCOVERY}

S.Z. Langer

Synthelabo Research, 31 avenue Paul-Vaillant Couturier

B.P. 110 - 92225 Bagneux Cedex, France.

There are many historical examples of CNS acting drugs which were discovered by serendipity and at the level of clinical observations in compounds which were developped for other indications. However, at present, several therapeutic classes of compounds exist which were developped following a biological rational and targetting either a neurotransmitter receptor subtype or an enzyme or a neuronal sodium-dependent transporter system. Examples of such targeted drug discoveries include : a) selective agonists of the $\mathrm{GABA}_{\mathrm{A}}$ receptor modulatory site (called $\mathrm{BDZ}$ or omega) as anxiolytic and hypnotic drugs ; b) inhibitors of the neuronal sodium-dependent serotonin transporter as anti depressants and for the treatment of obsessive compulsive disorders and c) selective, reversible inhibitors of monoamine oxydase type $A$ as antidepressants. With the development of molecular biology and the cloning and characterization of multiple neurotransmitter receptor subtypes, new and exciting possibilities are available for medicinal chemists in the search for selective agonists, partial agonists or antagonists for some of these receptors. Such compounds may offer novel therapeutic approaches with advantages over existing medications for several CNS diseases. In addition the successful therapeutic action of these selective compounds may serve to validate biological hypothesis of the pathophysiology of certain CNS diseases.

\section{ALTERNATIVE PROTOCOLS AND EVALUATION INSTRUMENTS}

Y. Lecrubicr

INSERM U 302 - Hôpital La Salpêtrière - 47, bd de l'Hôpital - 75013 Paris

The classification of psychotropics is entirely based on the effects of the first drigs available. These effects were defined according to the classification of patients used in the sixties. Although no relationship was found between the mechanism of action of drugs and biological modifications in patients this is still true, specially for regulatory agencies.

Due to our progresses in the knowledge of CNS an increasing number of drugs selectively interacting with newly discovered receptors or acting at other stages of neurotransmission are now available. These are no more "me too" drugs and the predictive value of pharmacological tests is uncertain. As a consequence numerous patients subgroups are potential targets for a large number of compounds for which the effective dosage is unknown. In parallel official or implicit guidelines favoured phase II protocols including large numbers of patients in a single, specific diagnostic area. New methodological approaches are urgently needed to avoid this unbearable situation.

A clinical preselection of new compounds is probably unavoidable.

- Are biological exams like PET able to help in dose finding, at what stage of development, in what patients ?

- Are brain functions specifically modified by new compounds good predictors of further therapeutic action. A research for clinical model should then be developed.

- Should syndromes better than diagnosis be considered to be valuable targets at an early stage (anhedonia + withdrawal, poor control of impulse. chronic worrying) ?

e.g. 1) Is a drug interacting with prefrontal DA receptors ? 2) What are the corresponding functions (motivation, anhedonia..) ? 3) Can this be assessed in normals or in patients showing such problems irrespective of diagnosis? in normals or in patients showing such problems irrespective of diagn
4 ) In case of positive response what are the more likely indications? When functional or dimensional testings are possible, normals or patients may be trained and an early response may be obtained on small population samples.

Since few is known for true innovating drugs early protocols should explore different clinical levels for potential therapeutic action:

e.g. Depression (axis I diagnosis) Avoidance (axis II diagnosis) pain or insomnia (symptomatic level) but eventually separation anxiety or interpersonal sensitivity (psychological traits).

Is the aim of early trials to prove a dose of a substance to be better than placebo or to prove that a dose range efficacy and tolerance can be identified with a high probability. The number of patients included and the number of placebo treated patients is very different in the second case.
NEGLECTED, BUT OF MAJOR CLINICAI RFLEVANCE: SUBJECTIVE EFFECTS OF NEUROLEPTICS D. Naber

Dept. of Psychiatry, University of Munich, Nussbaumstr. 7, D-80336 Munich, Germany

clinical trials of neuroleptics focus on acutely psychotic patients. However, wanted and unwanted effects of maintenance treatment differ markedly from those of short-term treatment and should be investigated more. One major problem is low compliance, explained by extrapyramidal motor side effects (EPMS) and reduced well-being, described as "pharmacogenic" or "akinetic depression".

A self-rating scale ( 38 items, 5 subfactors) was developed to measure subjective well-being under neuroleptics (SWN). Analyses indicate reliability (Cronbach's $\alpha .87-.91$ ) and practicability. Data from 230 remitted patients revealed that SWM correlated with negative symptoms (BPRS, PANSS), other self-ratings (POMS, SDS, Bf-S) and EPMS; but these variables explained only 19-478 of SWN variance. A repeated application after 3 months in 82 patients did not show any altered SwN in those with constant neuroleptic medication, but marked alterations, if dosage or drug was changed. SWN in 28 patients, negatively selected because of therapy-resistance or major side-effects, and therefore treated with clozapine, was significantly $(t=1.79, p=.03)$ better than in 38 patients under classical neuroleptics. Moreover, already at dismissal, patients who 4-6 months later were non-compliant ( $n=14)$, differed significantly in SWN, but not in BPRS/PANSS or POMS/SDS/Bf-S from those who remained compliant $(n=34)$.

These data indicate that $S W N$ is a useful tool to investigate a hitherto neglected dimension. Subjective effects of neuroleptics are of critical relevance regarding compliance. They are measurable and should be considered more in trials of neuroleptic drugs. 